\title{
Kongressbericht der 61. Jahrestagung der Deutschen Gesellschaft für Phlebologie vom 18.-21. September 2019 in Münster
}

Mit dem Kongressthema „Tradition und Innovation" fand die 61. Jahrestagung der Deutschen Gesellschaft für Phlebologie vom 18.-21. September 2019 in der Halle Münsterland in Münster mit dem Kongresspräsidenten Prof. Dr. med. Tobias Görge aus der Universitätshautklinik Münster statt.

Mit gut 1000 Teilnehmern, davon 150 Teilnehmer am Crashkurs und Update Phlebologie schon am Vor-Kongresstag, ausgebuchten Hands-On-Workshops und 45 ausstellenden Firmen sowie einem ansprechenden Rahmenprogramm war die Jahrestagung eine rundum gelungene Veranstaltung.

Das in den vergangenen Jahren bewährte Konzept des „aktiven wissenschaftlichen Beirats" fand auch in Münster wieder Anwendung. Die Organisation einzelner wissenschaftlicher Sitzungen in den jeweiligen Spezialgebieten der Vorsitzenden hat dazu beigetragen, dass ein breit gefächertes Programm gestaltet werden konnte. Mal waren die Sitzungen von Kurzreferaten geprägt, die gegeneinander bewusst provozierend die Diskussionen herausforderten, mal waren Sitzungen von Grundsatzreferaten gekennzeichnet, die den wissenschaftlich aktuellen Status quo eines Themas präsentiert sowie zu erwartende Innovationen skizziert haben.

Am Mittwoch machten der Crashkurs und das Phlebo-Update den Auftakt in bewährter Form, organisiert von Frau PD Dr. Felizitas Pannier und Prof. Dr. Stefanie ReichSchupke, denen wir an dieser Stelle einen großen Dank für ihr Engagement zollen. Wie in den letzten Jahren zeigte sich hier, dass gerade dieses Format viele neue und junge Gesichter anspricht. Die nachhaltigen Diskussionen, die verschiedenen interdisziplinären Blickwinkel und Sichtweisen, gerade auch aus der Praxis, bereichern beide Kurse immens.
Die Halle Münsterland gab der Tagung einen hellen Rahmen mit kurzen Wegen, der das Zusammenspiel von Fortbildungsblöcken und Kontaktaufnahmen in der Industrieausstellung förderte. Die Universitätsstadt Münster förderte den Trend für ein zunehmendes Interesse an der Phlebologie und untermauerte den Erfolg der Nachwuchsförderung, der sich erfreulicherweise nun schon seit vielen Jahren fortsetzt.

Im zweiten Jahr wurde das Phlebo-Start-up durchgeführt, das sich an Medizinstudenten richtet. Den interessierten angehenden Medizinern aus benachbarten Universitäten wurden Grundlagen der phlebologischen Diagnostik und Therapie vermittelt. Nach dem Startschuss 2018 hat sich die Teilnehmerzahl im Jahr 2019 fast verdoppelt. Ermöglicht wurde dieses neue Ausbildungsformat von der Firma Sigvaris.

Wieder eingeführt wurden am Donnerstag und Freitagvormittag Plenarsitzungen, am Donnerstag zum Thema „Hygiene in der Phlebologie“, am Freitag zum Thema „Das schöne Bein - kosmetische Aspekte in der Phlebologie“. Beide Sitzungen waren überdurchschnittlich gut besucht mit einer wunderbaren Mischung aus wissenschaftlicher Diskussion und Präsentation innovativer Betätigungsfelder für den Phlebologen.

Darüber hinaus wurden im Weiterbildungsforum, organisiert von Dominic Mühlberger und Jasmin Woitalla-Bruning, Grundlagen in Diagnostik und Therapie in der Phlebologie vermittelt sowie auch erstmals Abrechnungs- und berufspolitische Fragen ins Programm integriert.

Ein weiteres Highlight war die Verleihung der „Ratschow-Medaille“ an Dr. Werner Blättler am Samstag. In der Sitzung zum Thema „Subjektive venöse Symptome -
Welche Bedeutung haben sie in der Phlebologie?" behandelte sein Festvortrag das Thema „Venensymptome sind homöostatische Gefühle, nicht Krankheit“.

Die Ratschow-Gedächtnismedaille wird im Jahresrhythmus seit 1969 vom CURATORIUM ANGIOLOGIAE INTERNATIONALIS an besonders verdiente wissenschaftliche Persönlichkeiten aus dem Bereich Gefäßmedizin und diese tangierenden Disziplinen verliehen.

An allen 3 Kongresstagen waren die „ask the experts"-Sitzungen früh um 8 Uhr gut besucht. Die Möglichkeit, Experten zu allen Themen, die Phlebologen in der Praxis beschäftigen, persönlich zu befragen und vor allem über eigene Erfahrungen und Fälle zu diskutieren, wurde gut angenommen.

Parallel zum wissenschaftlichen Programm wurden für interessierte Teilnehmer zahlreiche Hands-on-Workshops, z. B. zum Erlernen endovenöser Techniken, angeboten. Neben den Klassikern Sklerotherapie und Duplexsonographie war auch ein Workshop zum Thema „Liposuktion und ästhetische Nahttechniken“ im Angebot.

Die Halle Münsterland war eine ideale Location mit kurzen Wegen und der Möglichkeit zu intensiven Dialogen sowohl in den Vortragssälen als auch in der Industrieausstellung.

Eine Neuerung in der Industrieausstellung war ein Diskussionsforum - am Donnerstag sprachen Prof. Dr. Markus Stücker und Prof. Dr. Tobias Görge über die aktuelle Leitlinie Kompressionstherapie, am Freitag stellte Dr. Volker Busche aus Regensburg das Thema „Gehirn unter Strom - Umgang mit dem digitalen Alltagsstress" in höchst unterhaltsamer und informativer Form vor. 


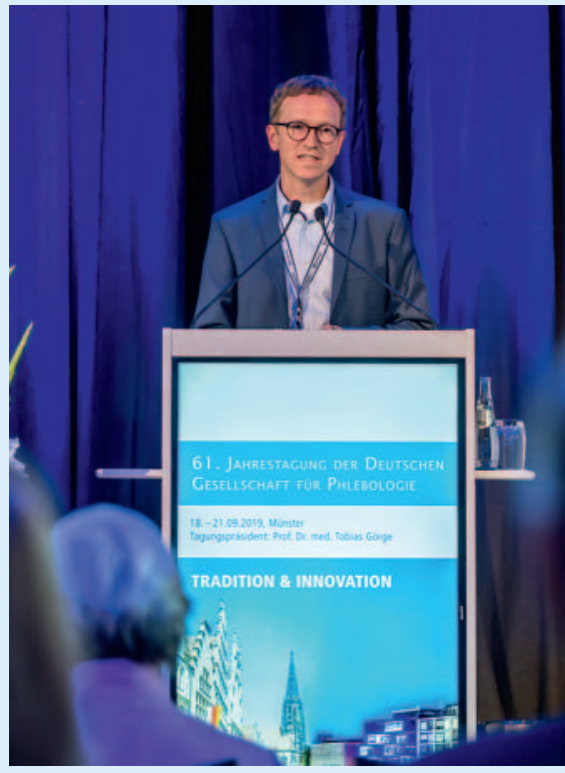

Eröffnung des Kongresses durch Prof. Görge.

Darüber hinaus wurde den täglichen Problemen des Praxisalltags reichlich Platz eingeräumt; so gab es wissenschaftliche Sitzungen zum Themenfeld Kompression, die Sklerosierungstherapie wurde behandelt und der große Raum der operativen und endoluminalen Therapie fand in zahlreichen Sitzungen Berücksichtigung. Venenerhaltende Therapiekonzepte bei der Stammvarikose konnten erläutert werden ebenso wie die Thrombosetherapie. In Sitzungen zum Thema „Der interessante Fall“ wurden Situationen aus dem Praxis- und Klinikalltag geschildert und ausführlich diskutiert.

45 ausstellende Firmen konnten die Innovationen in der fachgebundenen Medizintechnik und der Pharmakologie begleiten und so den Besuchern auch auf diesem Gebiet aktuelles Wissen vermitteln.

Wie immer stellte der Kongress die Plattform für Ehrungen und Preisverleihungen dar.

\section{Kreussler-Travel-Awards}

Thea Westphal, Greifswald: „Messung der gesundheitsbezogenen Lebensqualität bei CVI (TLQ-CVI): Einfluss eines MKS“

Olivia Danneil, St.-Josef-Hospital, Bochum: „Tiefe Muskelvenenthrombose nach Sklerosierung eines Seitenastes unter laufender Therapie mit Tamoxifen“

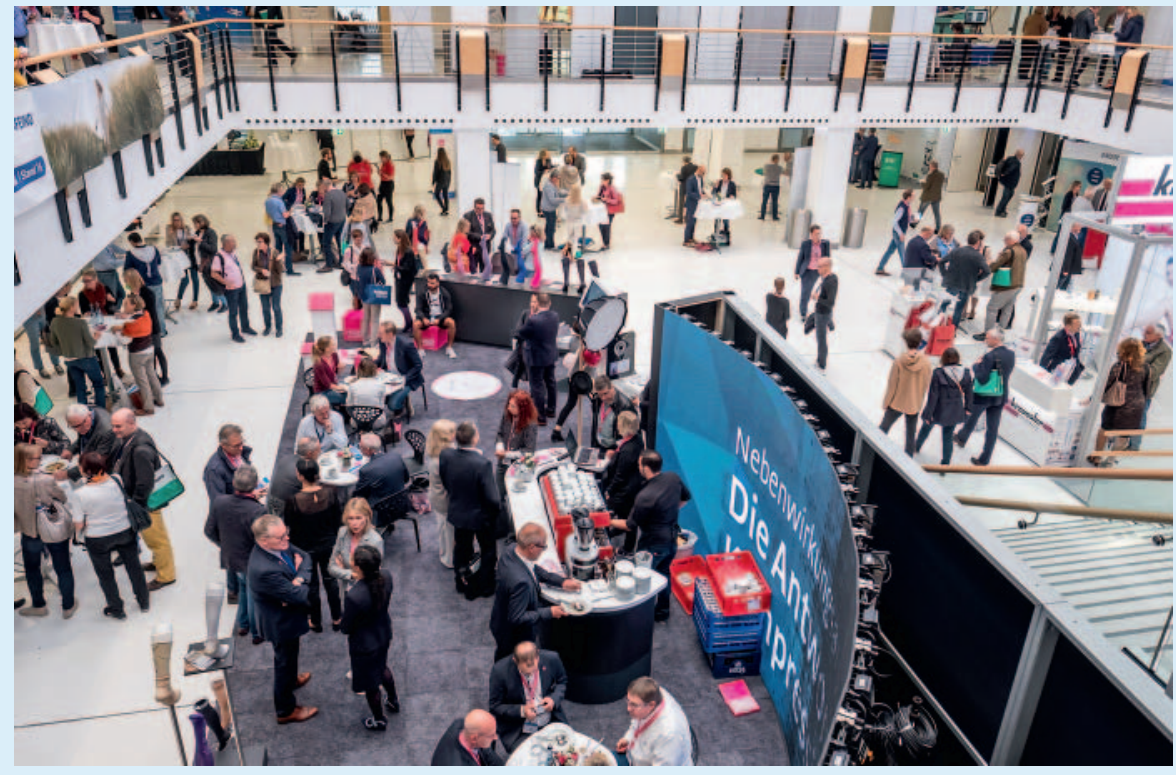

Impression aus der Industrieausstellung.

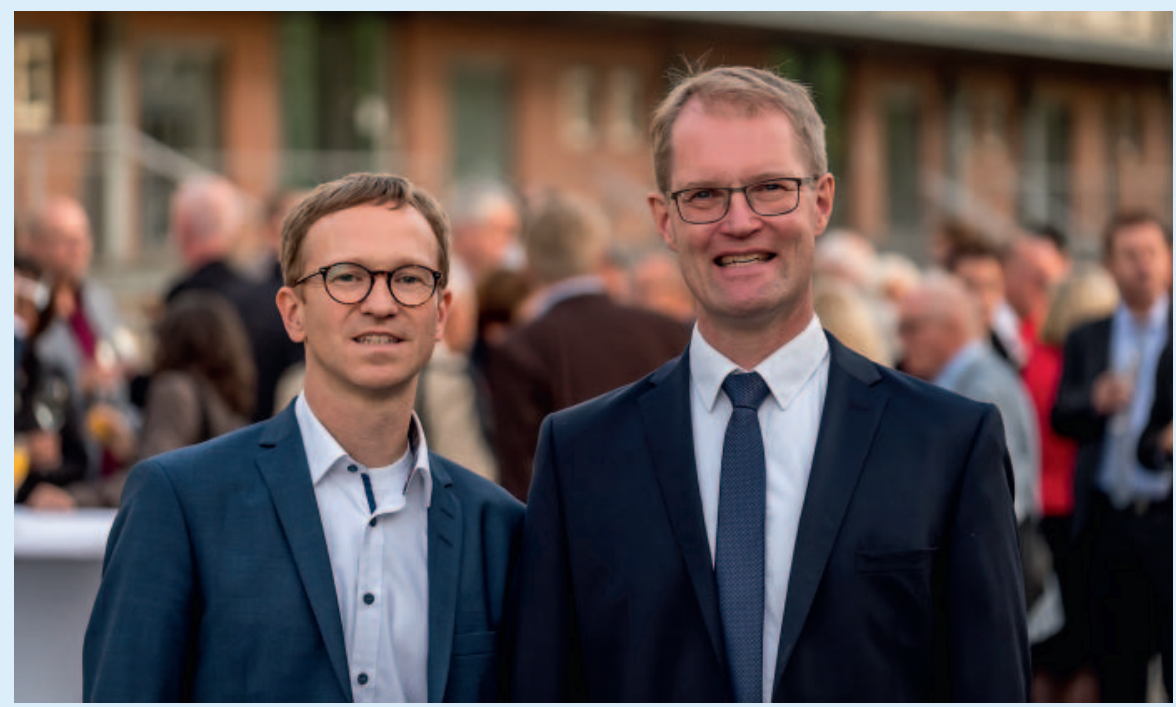

Kongresspräsident Prof. Görge und DGP-Präsident Prof. Stücker.

Bauerfeind-Doktorandenpreis

Carolin Mitschang, Universitätshautklinik Münster: „Die Lebensqualität von Patientinnen und Patienten mit diagnostizierter Stammveneninsuffizienz vor und nach Durchführung einer Radiofrequenzablation“ (betreut durch Prof. Görge)

Irene (Joana) Döll, Universitätsmedizin Greifswald - Dermatologie: „Evaluation von Akzeptanz und Nutzerzufriedenheit nach teledermatologischer Behandlung in der Zentralen Notaufnahme der Universität
Greifswald mithilfe des TeledermatologieSystems Mobil Skin ${ }^{\circledR “ ~(b e t r e u t ~ d u r c h ~ P r o f . ~}$ jünger)

\section{Juzo-Forschungspreis Phlebologie Jana Ziob, Bonn}

\section{SIGVARIS-Förderpreis Phlebologie}

Dr. med. Christine Fink, Heidelberg (3500,-€ für phlebologische Kongresse und Fortbildungen mit paralleler Berichterstattung) 
SIGVARIS-Studentenpreis Phlebologie

Hanna Brockhoff, Bochum, im 8. Semester (1000,-€ für phlebologische Fortbildungsveranstaltungen der Deutschen Gesellschaft mit paralleler Berichterstattung)

\section{Varady-Vortragspreis}

Zoltan Böhm, Frankfurt

\section{Medi-Reisestipendien}

In diesem Jahr wurden 15 Preise vergeben, die Auswahl und Bewerbung erfolgt direkt über die Firma Medi.
Am Freitagabend fand der Gesellschaftsabend im Speicher 10 in Münster, einer ehemaligen Heeresbäckerei, statt. Bei kollegialen Gesprächen und mitreißender Musik haben die Teilnehmer den Abend sehr genossen.

Der gesamte Kongress fand in einer lockeren, fröhlichen und gastfreundlichen Atmosphäre statt.

Zum Abschluss noch der herzliche Dank an alle, die zum Gelingen der 61. Jahrestagung der DGP beigetragen haben - dem wissen- schaftlichen Beirat, den aktiven Referenten und Vorsitzenden, der tollen Band am Festabend, der unterstützenden Industrie, ohne die eine solche Veranstaltung nicht möglich wäre, den Musikern des Rahmenprogramms, den vielen hilfsbereiten und freundlichen Hostessen, der Technik, der Kongressorganisation und dem zuarbeitenden Service und vor allem dem Kongresspräsidenten, Prof. Dr. Tobias Görge.

Nun schauen wir nach Leipzig - vom 2.-5.9.2020 freuen wir uns darauf, im Congress Center in Leipzig zu Gast zu sein. 